\title{
A DETECTION METHOD OF INTERSECTIONS FOR DETERMINING OVERLAPPING USING ACTIVE VISION
}

\author{
Pablo Gil, Fernando Torres \\ Department of Physics Engineering Systems and Signal Theory, University of Alicante, Alicante, Spain \\ pgil@dfists.ua.es, ftorres@dfists.ua.es
}

Oscar Reinoso

Department of Industrial Systems Engineering, Miguel Hernandez University, Elche, Spain

o.reinoso@umh.es

Keywords: $\quad$ Discontinuities, Overlapping, Occlusion, Occluded partially objects, Structured light.

\begin{abstract}
Sometimes, the presence of objects difficult the observation of other neighboring objects. This is because part of the surface of an object occludes partially the surface of another, increasing the complexitiy in the recognition process. Therefore, the information which is acquired from scene to describe the objects is often incomplete and depends a great deal on the view point of the observation. Thus, when any real scene is observed, the regions and the boundaries which delimit and dissociate objects from others are not perceived easily. In this paper, a method to discern objects from others, delimiting where the surface of each object begins and finishes is presented. Really, here, we look for detecting the overlapping and occlusion zones of two or more objects which interact among each other in a same scene. This is very useful, on the one hand, to distinguish some objects from others when the features like texture colour and geometric form are not sufficient to separate them with a segmentation process. On the other hand, it is also important to identify occluded zones without a previous knowledge of the type of objects which are wished to recognize. The proposed approach is based on the detection of occluded zones by means of structured light patterns projected on the object surfaces in a scene. These light patterns determine certain discontinuities of the beam projections when they hit against the surfaces becoming deformed themselves. So that, such discontinuities are taken like zones of boundary of occlusion candidate regions.
\end{abstract}

\section{INTRODUCTION}

Recognition of objects in image space is not a trivial problem. Commonly, in robotic environments, images are taken from scene where several objects interact together. In addition, the illumination conditions, view points, positions and orientations of the objects that are desired to identify can change at every moment of time.

In this context, the objects in image space suffer the presence of occlusions and shadows which make difficult or prevent the recognition due to the ambiguity of boundary between different object surfaces, especially when free-form surfaces are involved in the scene.

To avoid some concrete types of occlusions, active vision can be adopted. Structured light or pattern projection systems have been used for this purpose.

Our method is based on the assumption that the occlusions are not caused by lack of visibility due to shadows or by opacity of an object which hides part of itself due to its geometrical form. Here, we have assumed that the occlusion is caused by overlapping, with or without contact. On the one hand, we consider overlapping with contact when the surface of an object $A$ is in contact with the surface of another object $B$, and the surface of $A$ is partially hidden by the surface of B. And, on the other hand, overlapping without contact is considered when the spatial pose of each object at different depth levels (different distance from camera used like view point) cause that the pose of an object A prevents to perceive another possible B correctly, because B is 
in the background of the scene. In these practical applications, it is the camera and its relative pose which need to be online adjusted.

The objective of this paper is to find the intersection zone among surfaces of objects. Such intersection is caused by surfaces discontinuity among different objects. Determining this discontinuity, it is possible know which is the intersection zone that delimit a possible occlusion zone of an object hidden by another one.

This paper is organized as follows: The concept and the types of occlusion are described in section 2, also in this section we briefly expose the recognition systems for occluded object presents in the literature and which is the main problem of each system. Section 3, show a method based on structured light to detect occluded zones by overlapping between objects in the image space. Our approach consists of two steps. First, we fit the contours of beam projection in the image with polygonal approach. Second, it is commented a clustering process to separate beam projection over different surfaces or surfaces with different depth level, previously fit as polygonal contours. The presented clustering process combines moments and distances. To this end, our clusters are fit afterwards by means of straight lines. These one delimits the overlapping zone. Section 4 gives the experimental results in the implementation and 5 the conclusions.

\section{CONCEPT AND ANALYSIS OF OCLUSSIONS}

The recognition and classification systems of objects are based generally on the recognition from the extraction of characteristics and properties of the visible part of the objects (Bhanu, 2003)(Ulrich, 2001)(Ying, 2002). Therefore, the recognition systems employed for computer vision do not often work correctly when there are partially occluded objects in a scene. This is because different types of objects can be very similar if its visible part is only observed (Figure 1).

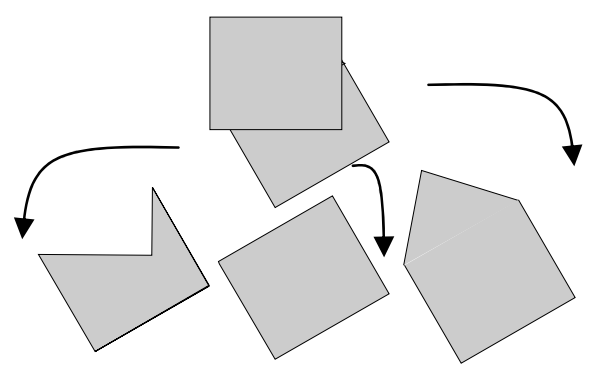

Figure 1: A same perception for different occluded objects.
There are different ways to classify the type of occlusion. Depending on the cause that produces it, the following categories can be distinguished:

- Occlusions in which an object covers a portion of the area of other object which is wished to be recognized and it is not absolutely visible. It is well-known like overlapping (Boshra, 2000)(Ying, 2002).

- Occlusions by opacity. An object hides part of itself due to its own geometry (Bhanu, 2003).

- Occlusions due to shadows. The kind of illumination causes shadows in the image around the object to be recognized or around the rest of present objects in the scene. Thus, an object can be occluded partially by its own shadow or the shadow of other object according to the kind of light employed (Bhanu, 2003)(Ulrich, 2001).

Almost all the recognition systems of occluded partially objects which obtain good results of success in the identification, are based on statistical or stochastics methods and need a great percentage of probability information (Park, 2003)(Ying, 2002).

Some of the techniques used in these systems aim at the recognition of flat objects, 2D-objects or three-dimensional ones (Chan, 2002) (Park, 2003)(Ulrich, 2001)(Ying, 2002) but whose registry in image space and the processing employed does not permit to work with the third dimension. In much of these works, is not important what type of occlusion appears, why it is caused or how it can be avoided or corrected or where is located that occlusion in the image. Only, the bi-dimensional information of the visible part of the object is analyzed and studied.

Here, a possible solution to improve the recognition of objects with occlusions is proposed. This one consists in determining where the occlusion is located in image space. Thus, if the occlusion zone is known, the camera can be repositioned with respect to a non-planar object given to avoid or reduce it. Furthermore, additional information about the boundary and occlusion zones of the objects can be important for a successful recognition process.

\section{DETECTION OF OVERLAPPING ZONES}

In this paper, we have proposed an approach for the detection of intersection in surfaces. When a light plane hit different surfaces, that is to say surfaces of a same object with different orientations or depth values, or hit surfaces of different objects, a 
discontinuity effect is caused in the projection planes. The discontinuity is caused by the breakage of light beam projection; thus several projection planes displaced in the space can be observed.

Our active vision system consist of a light pattern projector LASIRIS SNF, with a wavelength of $660 \mathrm{~nm}$, a power of $20 \mathrm{~mW}$, a fan angle about $15^{\circ}$ and 7 concentric circles with an interbeam angle of $0.77^{\circ}$, and a CCD camera. The intrinsic and extrinsic parameters of the camera are not necessary to know them to the overlapping detection, because reconstruction task are not made. Only, when the camera is wanted to reposition, both the intrinsic and extrinsic parameters of the camera are assumed from the pre-calibration offline.

\subsection{Approach of Contours}

In our work, the first stage consists of extracting the contours of circle pattern. To do it, firstly, a Gaussian smoothed process with a $3 \times 3$ size mask is done to reduce the produced noise when the beam hit too reflecting surfaces. Later, the image is binarized by means of a suitable threshold, according to the wavelength of the laser. Experimentally, a luminance threshold about 70 has been considered for $660 \mathrm{~nm}$. With this threshold it is possible to extract only the information of contours from the projected laser pattern.. Next, the projected circles in the image are detected by means of a convolution process. In this process, 8-connectivity masks are used (Teh, 1989). Thus, the pixels which determine each contour are found. This set of pixels corresponds to the 2D-points which compose each projected circle are fit by a polygonal approach.

The advantages of a polygonal approach are, on the one hand obtaining a simple representation for each contour which permits a fast and efficient comparison process between contours. On the other hand, we have the advantage of the reduction of the number of interest points detected during the contour extraction process. Thus, the beam projection is only represented by a minimum set of interest points without loss of stability in the contour extraction process (Figure 2).

Our approach method is based on the DouglasPeucker's algorithm (Douglas, 1973)(Hersberger, 1993). Where, each contour is approximated by a poly-line, $P_{s}$. If a contour is defined as a sequence of $n$ points so that $C_{s}=\left\{p_{1}, p_{2}, \ldots, p_{n}\right\}$, a poly-line $P_{s}$, can be defined as the union of 1-degree segments, where $l$ denotes each segment.

$$
P_{s}=\bigcup_{i=1}^{n-1}\left\{l_{i} l_{i+1}\right\}=\bigcup_{i=1}^{n-1}\left\{t p_{i}+(1-t) p_{i+1} / 0 \leq t \leq 1\right\}
$$

$$
P_{s}=\left\{p_{1} \ldots p_{n} / n \geq 2\right\}
$$

The adjustment is based on the representation of set of points by means of edge segments where the proximity of each point to the edge segment must be inferior to a tolerance factor $\varepsilon$ which is taken like reference threshold. The proximity is measured like a normal distance vector to each candidate edge segment.

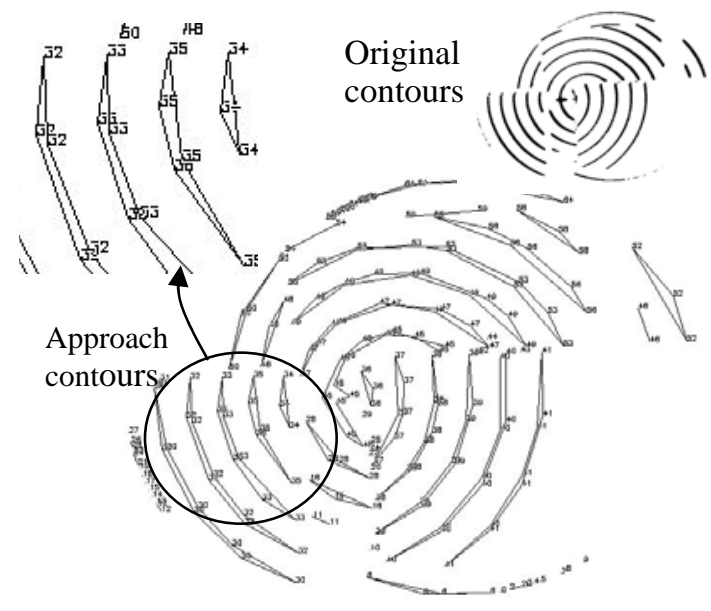

Figure 2: Approach of a beam.

The algorithm begins approximating all the points of a same contour, $C_{s}=\left\{p_{1}, p_{2}, \ldots, p_{n}\right\}$, with a poly-line which joins the fist and the last point of the edge contour, $P_{s}=l_{1}=\left\{p_{1} p_{n}\right\}$. The distance of each intermediate point to the poly-line is measured, and the farthest point with a tolerance greater than the $\varepsilon$ value, is added to the simplification process and takes part to build a new poly-line $P_{s}=\left\{p_{1} p_{i} p_{n}\right\}$. Later, this one is divided into two segments $l_{1}=\left\{p_{1} p_{i}\right\}$ and $l_{2}=\left\{p_{i} p_{n}\right\}$ and the union of both will compose as $P_{s}=\left\{l_{1}, l_{2}\right\}=\left\{p_{1} p_{i} p_{n}\right\}$.

Thus, these steps are repeated iteratively for each edge contour until all the points which belong to the poly-line do not violate the value rank marked by the tolerance factor. If the distance of an intermediate point to a poly-line is inferior to the tolerance factor, this one is not taken into account for the simplification process of the poly-line. The polygonal adjustment process has permit to approximate the laser beam contours in a more stable way than a set of contour points. Now, each edge contour is composed by an inferior number of points. Each contour is a set of points greater than two which it is the minimum number of points necessary to define a segment.

Nevertheless, in spite of the previous smoothing pre-processing, during the contour detection process, some contours have been approximated by poly- 
lines of small length, this polylines are formed by a small number of points. This fact can be due to noise, contour points which contribute with little information because it represents very small and despicable discontinuities. Therefore, the approximated contours should be filtered in order to work only with those having some length and are determined by a number of points greater than three $P_{s}=\left\{p_{1} p_{2} \ldots p_{n} / n \geq 3\right\}$. Once filtrated, it is necessary number the candidate contours again with a new numberation, despising those which are not useful because they do not contribute with important discontinuity information or because they can cause confusion.

\subsection{Clustering and approach of discontinuity}

Once, the polygonal approaches have been filtered, the end points are only chosen. These points are the tactically important points which will determine the directions of discontinuity $P_{s}=\left\{p_{1} p_{n} / n \geq 3\right\}$. These directions of discontinuity are not but the zones of breakage of the light beam projections, when the beam hit over two surfaces with different depth or orientation. Later, a method of adjustment of the tactically important points is applied by means of straight lines. These straight lines determine the candidate zones and the suitable direction for the search of possible discontinuities which are present in the changes of surface (Figure 3).

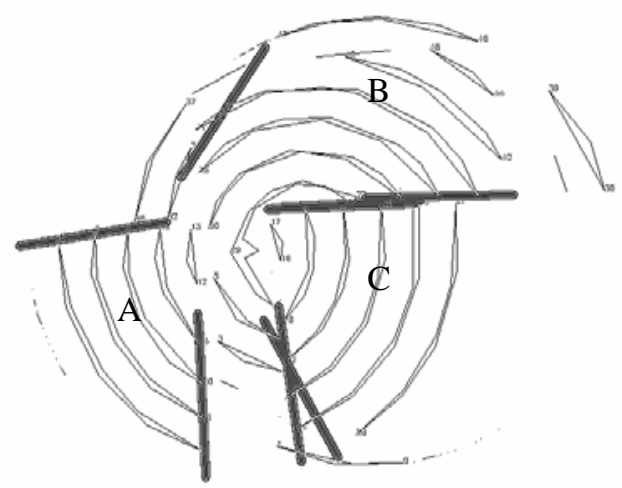

Figure 3: Intersection and discontinuity zones among three objects.

A clustering process of tactically important points is carried out to be able to determine whichever straight lines are necessary to fit them, and to discrimante what tactically important points belong to one or intersection. The clustering process permits to group the tactically important points according to two parameters: inertial moments
(Chavez, 1999) and metric distances (Teague, 1980). Thus, each edge contour approximated by a polyline $P_{S}$ has two tactically important points, each one must belong to different clusters, and must have associated the central moments of the poly-line to which they belong. The central moments are invariant to transfering identical beams in the image, and they do not depend on the position that the beam projections have in the image.

$$
\mu_{p, q}=\iint\left(x-x_{c}\right)^{p}\left(y-y_{c}\right)^{q} f(x, y) d x d y
$$

Furthermore, it is possible to determine the rotation of each edge contour around its gravity centre from the central moments and with the aid of the components of inertial tensor, as:

$$
\theta=\frac{1}{2} \arctan \frac{2 \mu_{1,1}}{\mu_{2,0}-\mu_{0,2}}
$$

The parameter of inertial moments is employed to discern between tactically important points which belong to poly-lines, and therefore to contours with the same orientation. This way, we have supposed that the beam projections over the same surface of an object $A$ have similar inertial moments. Although, in an object $B$ which is overlapping and occluding part of surface of $A$, the inertial moments estimated from the beam projections over a surface of $B$, are different to those estimated from $A$ (Figure 3 ). When a discontinuity is detected an orientation change of the beam projections takes places, and consequently a new value of the inertial moments.

Also, another parameter to emphasize is the distance of Minkowski $L_{2}$. Thus, the distance of each tactically important point to the rest of tactically important points is calculated, trying to minimize it.

$$
d_{L_{r}}=\left(\sum_{i, j}\left|p_{i}-p_{j}\right|^{r}\right)^{1 / r}
$$

The distance parameter is used to avoid to group as points of a same discontinuity, those points which can agree at inertial moment, but due to their little proximity they may not belong to the same candidate zone. For this reason, only the tactically important points, near among them and whose polylines have similar inertial moments, are clusterized.

Then, we obtain $n$ sets of points of the following type: $p_{i}=\left\{s_{1} s_{2} \ldots s_{n}\right\} / 0<i<n$ where $\left\{s_{1} s_{2} \ldots s_{n}\right\}$ are the points nearer $p_{i}$ ordered according to proximity. Finally, the difference of moments for each set of points is obtained as it was mentioned 
above. From the distances and the difference of moments calculated for each set $p_{i}=\left\{s_{1} s_{2} \ldots s_{n}\right\}$, the clustering process is made. In this way, for any two points $p_{i}$ and $s_{j}$, these will be able to be jointly stored and included in the same cluster, solely if they fulfill that $d \theta_{i j}<\varphi_{c}$, with $\varphi_{c}$ the angular tolerance allowed to consider it similar orientation.

$$
d \theta_{i j}=\left|\theta_{p i}-\theta_{s j}\right|
$$

Finally, after the tactically important points have been grouped by means of the clustering process, (Figure 4), the candidate zones which represent discontinuities in the image are defined as those zones which cotain each cluster. In order to determine the search direction of those candidate zones and their boundary, it is necessary to make a linear adjustment by least-squares method which calculates the straight line segments which diminish the Minkowski's distance $\mathrm{L}_{1}-\mathrm{L}_{2}$ of each set of tactically importan points to the straight line segment (Figure 3).

In Figure 4, the clustered sets of points are represented by different colours.

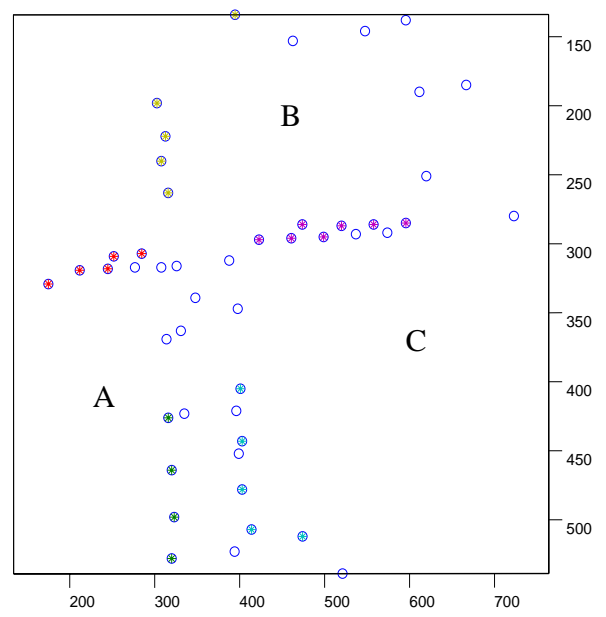

Figure 4: 2D-Representation of the clustering process.

The 2D-representation shows the relation of distance among the points of each cluster. Furthermore, in the distribution space of clusters shown in Figure 5, it is observed that using jointly parameters like distances and inertial moments, points which could be considered very different can be grouped. Thus, figure 5 shows the relation between the pose of each point in image space and its inertial moment.

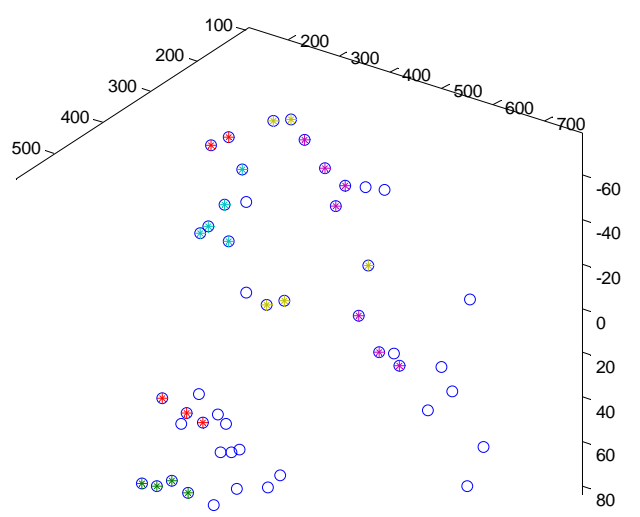

Figure 5: 3D-Representation of the clustering process.

zones between two objects. Thus, figures 4 and 5 represent the clusters used for the approach shown in figure 3 .

\section{EXPERIMENTAL RESULTS}

In our experiments, the objects used are polyhedrons. We have not considered objects with smooth surfaces. The size of captured image is 768x576 and all images have been acquired with a monochrome CCD in our laboratory.

The experimental results exhibit satisfactory coherence in the spatial detection by discontinuities when the discontinuities are caused by overlapping (Figures 6b and 6c). However, the proposed method works correctly and it is valid when the discontinuities are caused by contact between sides of two very near objects (Figure 6a).

Figure 6 shows the application of the proposed method on the detection of intersections among objects. In our method, the intersections are well detected while other type of discontinuities is not detected because they are not caused by the intersection between objects. In Figure 6a, two objects have a side of contact and therefore a discontinuity is caused by the contact between both. In Figures 6b, and 6c, there is an overlapping between both objects. This overlapping is caused because an object called $\mathrm{B}$ is located upon another one A. Moreover, there is an occlusion because B hidden part of surface of A. In theses two cases, the discontinuities caused by overlapping are only detected. 


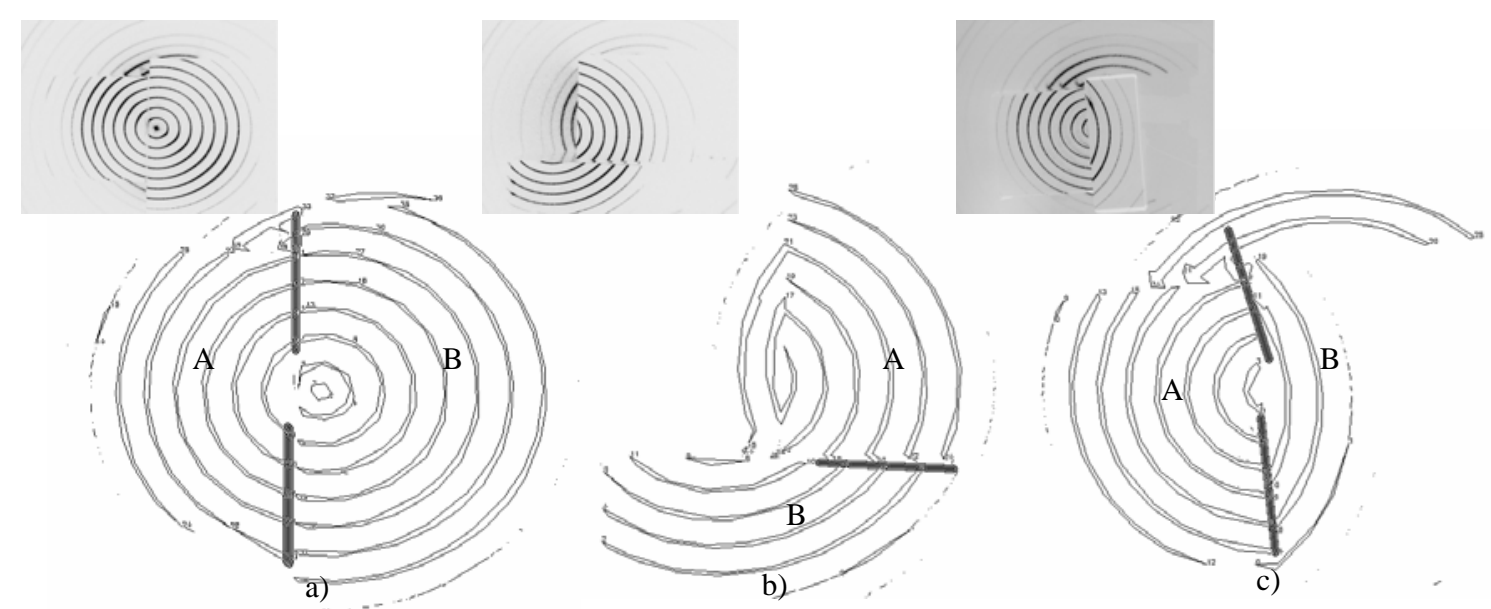

Figure 6: Results for different pose of objects (real objects in part superior and image processed in part inferior).

\section{CONCLUSION}

In this paper, we studied the occlusion by overlapping using concentric pattern projections. The developed method was implemented with satisfactory experimental results. The system can work well for detection of simple polyhedral surfaces without requiring apparent features. In the practice this system is used jointly with colour segmentation techniques. This active vision system is useful for occlusion detection when there are several objects in the scene with a same colour. In this case, the colour segmentation techniques fail. In addition, it is also useful to provide additional information when they work well, this is when the objects have colour different. Perhaps, an analysis of the proposed method could suggest that a method based on range data analysis could be applied to obtain discontinuities from depth information of a more robust way. However the computation of depth data requires a previous camera calibration, and therefore a triangulation method to calculate the 3dspace coordinates. The method proposed permits to work with cameras with unknown intrinsic and extrinsic parameters as opposed to the methods based on range data. In many recognition applications, in which the camera changes the localization and focal length, the use of the proposed method is an advantage because a recalibration of laser-camera system is not a necessary.

\section{REFERENCES}

Bhanu B., Lin Y., 2003. Stochastic models for recognition of occluded target. Pattern Recognition 36, pp. 28552873.
Boshra M., Ismael M.A., 2000. Recognition of Occluded Polyhedra from range images. Pattern Recognition 33, pp. 1351-1367.

Chan C.J., Chen S.Y., 2002. Recognizing Partially Occluded Objects Using Markov Model. International Journal of Pattern Recognition and Artificial Intelligence 16(2), pp. 161-191.

Chavez, E., Navarro G., Baeza-Yates R., Marroquín J., 1999. Searching in metric spaces.

Technical Report TR/DCC-99-3, Dept. of Computer Science, Univ. of Chile.

Douglas D., Peucker T., 1973. Algorithms for the reduction of the number points required to represent a digitized line or its caricature. The Canadian Cartographer 10(2), pp. 112-122.

Hershberger J., Snoeyink J., 1993. Speeding Up the Douglas-Peucker Line-Simplification Algorithm. Proc. $5^{\text {th }}$ Symp. On Data Handling, pp. 134-143.

Teague M.R., 1980. Image Análisis via the general theory of moments. Journal of Optical Society of America 70(8), pp. 920-930.

Teh C.H., Chin R.T., 1989. On the detection of dominant points on digital curves. IEEE Trans. PAMI 1(8), pp. 859-872.

Park B.G., Lee K.M., Lee S.U., Lee, J.H., 2003. Recognition of partially occluded objects using probabilistic ARG (attributed relational graph)-based matching. Computer Vision and Image Undesrstanding 90, pp. 217-241.

Ulrich M., Steger C., 2001. Empirical Performance Evaluation of Object Recognition Methods. Empirical Evaluation Methods in Computer Vision. IEEE Computer Society Press. Los Alamitos, C.A., pp. 6276.

Ying Z., Castañon D., 2002. Partially Occluded Object Recognition Using Statistical Models. International Journal of Computer Vision 49(1), pp. 57-58. 